\title{
Implementation of preemptive fluid strategy as a bundle to prevent fluid overload in children with acute respiratory distress syndrome and sepsis
}

\author{
Franco Díaz ${ }^{1,2,3} \mathbb{D}$, María José Nuñez ${ }^{1}$, Pablo Pino ${ }^{1}$, Benjamín Erranz ${ }^{3}$ and Pablo Cruces ${ }^{4,5^{*}}$
}

\begin{abstract}
Background: Fluid overload (FO) is associated with unfavorable outcomes in critically ill children. Clinicians are encouraged to avoid FO; however, strategies to avoid FO are not well-described in pediatrics. Our aim was to implement a bundle strategy to prevent FO in children with sepsis and PARDS and to compare the outcomes with a historical cohort.

Methods: A quality improvement initiative, known as preemptive fluid strategy (PFS) was implemented to prevent early FO, in a 12-bed general PICU. Infants on mechanical ventilation (MV) fulfilling pARDS and sepsis criteria were prospectively recruited. For comparison, data from a historical cohort from 2015, with the same inclusion and exclusion criteria, was retrospectively reviewed. The PFS bundle consisted of 1. maintenance of intravenous fluids (MIVF) at 50\% of requirements; 2 . drug volume reduction; 3 . dynamic monitoring of preload markers to determine the need for fluid bolus administration; 4. early use of diuretics; and 5. early initiation of enteral feeds. The historical cohort treatment, the standard fluid strategy (SFS), were based on physician preferences. Peak fluid overload (PFO) was the primary outcome. PFO was defined as the highest FO during the first $72 \mathrm{~h}$. FO was calculated as (cumulative fluid input - cumulative output)/kg*100. Fluid input/output were registered every $12 \mathrm{~h}$ for $72 \mathrm{~h}$.
\end{abstract}

Results: Thirty-seven patients were included in the PFS group (54\% male, 6 mo (IQR 2,11)) and 39 with SFS (64\%male, 3 mo (IQR1,7)). PFO was lower in PFS (6.31\% [IQR4.4-10]) compared to SFS (12\% [IQR8.4-15.8]). FO was lower in PFS compared to CFS as early as $12 \mathrm{~h}$ after admission [2.4(1.4,3.7) $\mathrm{v} / \mathrm{s} 4.3(1.5,5.5), p<0.01]$ and maintained during the study. These differences were due to less fluid input (MIVF and fluid boluses). There were no differences in the renal function test. PRBC requirements were lower during the first $24 \mathrm{~h}$ in the PFS (5\%) compared to SFS $(28 \%, p<0.05)$. MV duration was $81 \mathrm{~h}(58,98)$ in PFS and $118 \mathrm{~h}(85154)$ in SFS $(p<0.05)$. PICU LOS in PFS was $5(4,7)$ and in SFS was $8(6,10)$ days.

Conclusion: Implementation of a bundle to prevent FO in children on MV with PARDS and sepsis resulted in less PFO. We observed a decrease in MV duration and PICU LOS. Future studies are needed to address if PFS might have a positive impact on health outcomes.

Keywords: Fluid overload, Pediatrics, Mechanical ventilation, Sepsis, PARDS

\footnotetext{
* Correspondence: pcrucesr@gmail.com

${ }^{4}$ Pediatric Intensive Care Unit, Hospital El Carmen de Maipú, Santiago, Chile

${ }^{5}$ Centro de Investigación de Medicina Veterinaria, Escuela de Medicina

Veterinaria, Facultad de Ciencias de la Vida, Universidad Andrés Bello, Avda.

Republica 217, Santiago, Chile

Full list of author information is available at the end of the article
}

(c) The Author(s). 2018 Open Access This article is distributed under the terms of the Creative Commons Attribution 4.0 International License (http://creativecommons.org/licenses/by/4.0/), which permits unrestricted use, distribution, and reproduction in any medium, provided you give appropriate credit to the original author(s) and the source, provide a link to the Creative Commons license, and indicate if changes were made. The Creative Commons Public Domain Dedication waiver (http://creativecommons.org/publicdomain/zero/1.0/) applies to the data made available in this article, unless otherwise stated. 


\section{Background}

During the last two decades, intravenous fluid administration has been the cornerstone of treatment of children with hemodynamic instability. Restoration of circulating blood volume and perfusion to tissues is the primary end point of this therapy, decreasing mortality and morbidity of critically ill children with clinical signs of poor perfusion and shock [1]. Supported by guidelines and protocols, rapid administration of intravenous fluid is currently one of the most frequent interventions in critical care [2-5]. Intravenous (IV) fluid resuscitation may be lifesaving, but many studies have found that positive fluid balance is associated with negative outcomes in many clinical scenarios [6-15]. Critically ill patients are especially prone to positive fluid balance due to excessive fluid input (resuscitation fluids, maintenance intravenous fluid, continuous drug infusions, blood products and IV treatments), limited elimination of fluids (due to counterregulatory mechanisms such as antidiuretic hormone secretion) and due to capillary leakage in the interstitium, resulting in organ edema and dysfunction [16-19]. Detrimental effects are very pronounced in organs with a high density of capillaries, such as the lung, due to capillary leakage associated with a high hydrostatic pressure [16, 19-21]. Fluid overload has been associated with pulmonary dysfunction, hypoxia, longer duration of mechanical ventilation and ICU stay in children and adults on mechanical ventilation (MV) $[6,7,13,15,20,22-24]$. In contrast, in the setting of acute respiratory distress syndrome (ARDS), diuretic use and restrictive fluid management are associated with lower mortality and faster liberation from MV, respectively [18, 24-26].

Despite these overwhelming data, few studies in pediatrics have addressed how to avoid significant fluid overload in patients under MV.

The objective of this study was to describe the implementation of a preemptive fluid strategy in children with sepsis and ARDS. In addition, a comparison with an historical cohort of infants was made.

\section{Methods}

Expedited review by the IRB approved the quality improvement project, waiving the requirement for a written consent.

Setting: During a 12-month period (January to December 2016), all children admitted to the Pediatric Intensive Care Unit at Hospital Padre Hurtado were screened. Our unit is a 12-bed general medical and surgical PICU and does not take care of patients after congenital heart surgery or those receiving a transplant.

Participants: All patients younger than 24-months who received MV and fulfilled the pediatric ARDS (pARDS) and sepsis criteria, were prospectively identified. pARDS was defined according to the PALICC criteria [27] and sepsis was defined according to the International Pediatric Sepsis Consensus Conference definitions [28]. Patients were excluded if they were younger than 28 days old, had chronic renal insufficiency or end-stage renal disease, required dialysis, had a cyanotic congenital heart disease, underwent tracheostomy or required the chronic use of a positive pressure ventilation system.

\section{Preemptive fluid strategy (PFS)}

As previously reported [29], this protocol was developed following 5 principles, in the post-resuscitation phase of critical illness:

1. Maintenance of intravenous fluids (MIVF) at $50 \%$ of baseline requirements estimated by the HollidaySegar formula, ensuring adequate glucose infusion rate for normoglycemia.

2. Preparation of continuous infusions of medications (sedation and vasoactive drugs) and intravenous treatments (e.g., antibiotics) concentrated to the minimum volume recommended. Use of miniaturized devices for hemodynamic monitoring (i.e., pressure transducers) at the minimum rate of saline infusion.

3. Use of dynamic preload markers (pulse pressure variation) in addition to clinical assessment to decide administration of fluid boluses and early titration of vasoactive drugs.

4. Consideration of early use of diuretics when hypovolemia was ruled out, resuscitation goals were met and urinary output was less than $0.5 \mathrm{ml} / \mathrm{kg} / \mathrm{h}$.

5. Early initiation of enteral feeds.

A Historical cohort data was collected retrospectively with the same inclusion/exclusion criteria from June 2014 to December 2015. These patients were treated with standard fluid strategy and compared with the prospectively collected data of the patients managed with PFS.

\section{Standard fluid strategy (SFS)}

Standard fluid strategy can be summarized as:

1. Resuscitation phase: hemodynamic resuscitation based on Surviving Sepsis Campaign [2, 3].

2. Stabilization phase: patients received MIVF at $100 \%$ of baseline requirements estimated by the HollidaySegar formula.

3. Depletive phase: Initiation of diuretics after meeting resuscitation end points, hemodynamic stability and absence of any marker of dysoxia.

\section{Data collection}

Demographic and clinical data were recorded in a relational database. Fluid intake and output (I/O) were recorded every 
$12 \mathrm{~h}$ during the first 3 days after admission. To determine the causes of fluid overload and variations in clinical practice after initiation of the protocol, fluid intake was divided into MIVF, fluid bolus, enteral feeds, drug administration (e.g., antibiotics) and packed red blood cells (PRBC) transfusions. Renal function was monitored daily and electrolyte alterations were monitored every $12 \mathrm{~h}$ or more frequently depending on the treating physician's assessment. Urinary output and diuretic use were also recorded.

Vasoactive drug (VAD) support was recorded and maximum vasoactive inotropic score (VIS) score was calculated daily [30]. The percent of fluid overload (FO) was calculated using the following formula: [(total fluid intake (L) - total fluid output in liters (L)) / (admission weight in kilograms)*100] [31]. Peak FO was defined as the maximum percentage of FO during the first $72 \mathrm{~h}$ after initiation of invasive MV. No data were collected before PICU admission.

The primary outcome was to measure peak fluid overload (PFO). Fluid input and output were recorded as secondary outcomes to determine the causes of FO. Other clinical outcomes such as duration of MV, hospital and PICU length of stay (LOS) were recorded.

\section{Statistical considerations}

Descriptive statistics were used to summarize all continuous and categorical variables. Comparisons between patient groups were performed using Fisher's exact test for categorical variables and the Mann-Whitney $U$ test for continuous variables because of concerns about the normality of the distribution of these variables. Two-way repeated measures ANOVA test was performed for comparisons (fluid overload) among and between groups during the study period. All statistical tests were 2-sided and were performed with a $P$-value less than 0.05 indicating statistical significance. The SPSS software package (version 20.0; SPSS, Chicago, IL, USA) was used for the statistical analyses.
Table 1 Demographics and clinical outcomes of septic children with ARDS on preemptive fluid strategy and standard fluid strategy

\begin{tabular}{lll}
\hline & Preemptive & Conservative \\
$\mathrm{N}$ & 37 & 39 \\
\hline Male & $54 \%$ & $64 \%$ \\
Age (mo) & $6(2,11)^{*}$ & $3(1,7)$ \\
Weight (kg) & $8.4(5.4,10)^{*}$ & $5(4.7,9)$ \\
Comorbility & $38 \%$ & $49 \%$ \\
RSV & $54 \%$ & $51 \%$ \\
P/F ratio at admission & $207(146,249)$ & $193(146,234)$ \\
Duration of MV (h) & $81(58,98)^{*}$ & $118(85.5154 .5)$ \\
PICU LOS (days) & $5(4,7)^{*}$ & $8(6,10)$
\end{tabular}

$\left({ }^{*} P<0.05\right)$

Abbreviations: $A R D S$ acute respiratory distress syndrome, $R S V$ respiratory syncytial virus; $P / F$ ratio $\mathrm{PaO}_{2} / \mathrm{FiO}_{2}$ ratio, $M V$ mechanical ventilation, $P I C U$ LOS pediatric intensive care unit length of stay

\section{Results}

Thirty-seven patients were included in the PFS group and compared with 39 patients in the SFS group (Table 1). No mortality was noted in this cohort.

Fluid overload was significantly lower in the PFS group compared to the SFS group at 12, 24, 36, 48, 60 and $72 \mathrm{~h}(P<0.05)$ (Fig. 1). PFO was significantly lower in the PFS group (6.31\% [IQR 4.4-10]) compared to the SFS group (12\% [IQR 8.4-15.8]).

The PFS group required significantly less fluid input due to less MIVF and fewer resuscitation boluses compared to SFS (Fig. 2a and b). The PFS group had a lower urinary output, but renal function tests were not different from the SFS group. Diuretic use was frequent in both groups, at $24 \mathrm{~h} 54 \%$ of patients on PFS received at least one diuretic bolus and $15 \%$ of patients on SFS $(P=0.003)$. The SFS group required more frequent continuous infusions of diuretics at 48 and $72 \mathrm{~h}$. (Table 2).

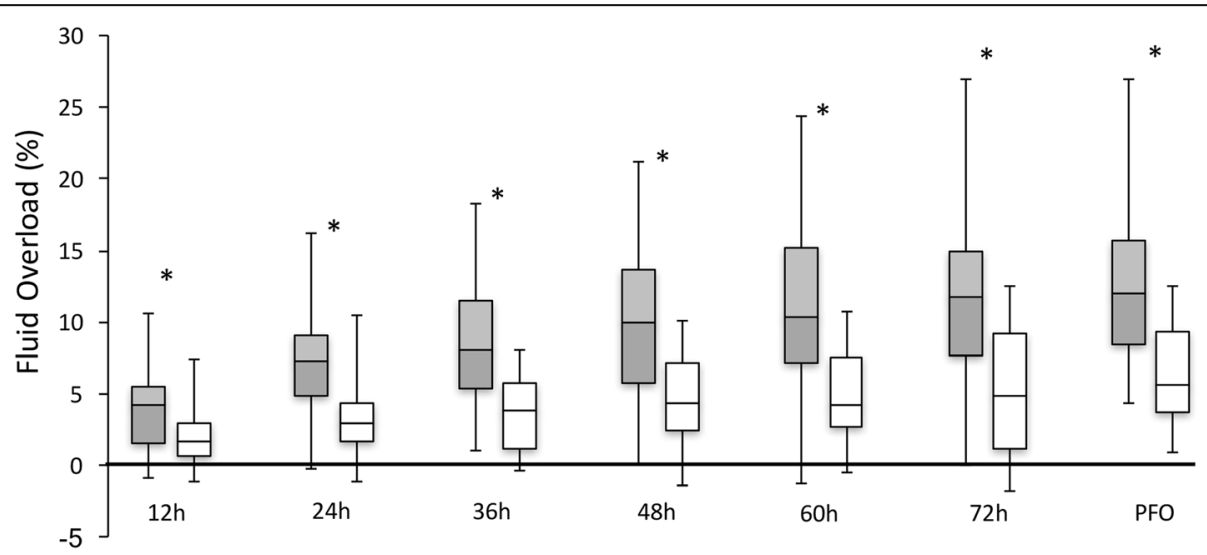

Fig. 1 Box plot graph of cumulative and peak fluid overload during the first $72 \mathrm{~h}$ after admission in children with sepsis and ARDS with preemptive fluid strategy (white) and standard fluid strategy (gray). (* $P<0.05$ ). Abbreviations: ARDS: acute respiratory distress syndrome; PFO: peak fluid overload 


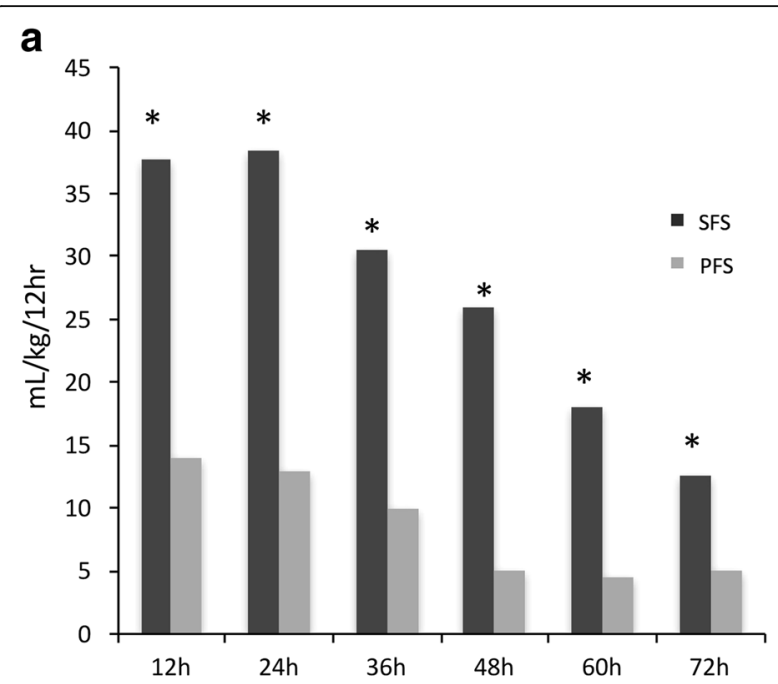

b

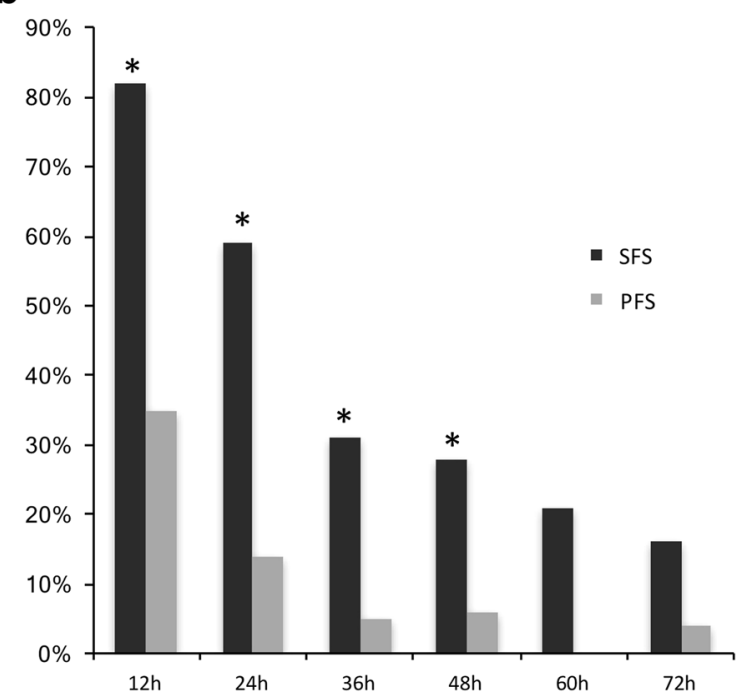

Fig. 2 Maintenance intravenous fluid administration $\left(\mathrm{ml} \cdot \mathrm{kg}^{-1} \cdot 12 \mathrm{~h}^{-1}\right)$ in both groups during the study intervals after admission (a). Percentage of patients that received at least one fluid bolus during the study intervals after admission (b). $\left({ }^{*} \mathrm{P}<0.05\right)$. Abbreviations: PFS: preemptive fluid strategy; SFS: standard fluid strategy
PRBC transfusions were less frequent in the first $12 \mathrm{~h}$ after admission in the PFS group. Enteral feeds were started earlier in the PFS group (Table 3). The median duration of vasoactive drug support was 2 days $(0,3)$ in the PFS group and 3 days $[2,4]$ in the SFS group $(P=0.018)$ and VIS was significantly lower during the first and the third day of admission in the PFS group. Use of greater than or equal to 2 vasoactive drugs was more frequent in the SFS group during the third day after admission (Table 3). Epinephrine support was more frequent in the SFS group during the first day after admission, whereas norepinephrine was more frequent during the third day after admission in the same group (Additional file 1: Figure S1).

Time to successful extubation was $81.5 \mathrm{~h}$ [IQR $56.5-$ 100.5 ] in the PFS group and $118 \mathrm{~h}$ [IQR 85.5-154.5] in the SFS group $(P<0.01$ by the log rank test). Additionally, PICU LOS was shorter in the PFS group compared to the SFS group (5 days [IQR 4-7] vs 8 days [IQR 6-10], $P<0.01)$.

\section{Discussion}

The main finding of our study is that prevention of fluid overload as a bundle for critically ill children was successfully implemented in a general PICU. Critically ill children with sepsis and pARDS that underwent preemptive fluid strategy had less peak fluid overload compared with standard fluid strategy. These differences were due to a lower requirement for fluid input from MIVF and fluid bolus resuscitation, especially during the first $48 \mathrm{~h}$. No detrimental effects were found on renal function test, urinary output or vasoactive drugs requirements.

The detrimental effects of fluid overload in critically ill children have been extensively reported in many different settings $[6-15,20-23]$, but this study is the first one to offer an alternative approach to avoid early fluid overload. We developed this bundle based on preliminary data (29) that showed that excessive fluid administration during the first $72 \mathrm{~h}$ after admission was the main responsible factor for early fluid overload during the course of critical illness.

Table 2 Renal function test, urinary output and percentage of patients that received diuretics in the preemptive fluid strategy and standard fluid strategy groups

\begin{tabular}{|c|c|c|c|c|c|c|}
\hline \multirow[b]{3}{*}{$N$} & \multicolumn{2}{|l|}{$24 \mathrm{~h}$} & \multicolumn{2}{|l|}{$48 \mathrm{~h}$} & \multicolumn{2}{|l|}{$72 \mathrm{~h}$} \\
\hline & PFS & SFS & PFS & SFS & PFS & SFS \\
\hline & 37 & 39 & 36 & 39 & 24 & 37 \\
\hline$\overline{B U N}\left(m g \cdot d L^{-1}\right)$ & $7(6,9.5)$ & $4(3,8)$ & $5(3,8)$ & $3(2,6)$ & $7(5,16)$ & $4(2,8.5)$ \\
\hline Creatinine $\left(\mathrm{mg} \cdot \mathrm{dL}^{-1}\right)$ & $0.22(0.16,0.28)$ & $0.18(0.14,0.24)$ & $0.21(0.19,0.3)$ & $0.2(0.15,0.26)$ & $0.22(0.14,0.35)$ & $0.22(0.19,0.36)$ \\
\hline Urinary output $\left(\mathrm{mL} \cdot \mathrm{kg}^{-1} \cdot \mathrm{h}^{-1}\right)$ & $1.8^{*}(1.1,2.8)$ & $2.9(1.8,3.7)$ & $2.3^{*}(1.6,3.5)$ & $4(2.9,5.7)$ & $2.6^{*}(2.1,3.75)$ & $4.1(3,5.2)$ \\
\hline Diuretic bolus (\%) & $54^{*}$ & 15 & 72 & 64 & 57 & 76 \\
\hline Continuous infusion of diuretic (\%) & 11 & 13 & $19^{*}$ & 59 & $17^{*}$ & 70 \\
\hline
\end{tabular}

$\left({ }^{*} \mathrm{P}<0.05\right)$

Abbreviations: PFS preemptive fluid strategy, SFS standard fluid strategy 
Table 3 Percentage of patients of each group that received packed red blood cell transfusion, enteral feeds and 2 or more vasoactive drugs at different study points

\begin{tabular}{|c|c|c|c|c|c|c|c|c|c|c|c|c|}
\hline \multirow[b]{3}{*}{ (N) } & \multicolumn{2}{|l|}{$12 \mathrm{~h}$} & \multicolumn{2}{|l|}{$24 \mathrm{~h}$} & \multicolumn{2}{|l|}{$36 \mathrm{~h}$} & \multicolumn{2}{|l|}{$48 \mathrm{~h}$} & \multicolumn{2}{|l|}{$60 \mathrm{~h}$} & \multicolumn{2}{|l|}{$72 \mathrm{~h}$} \\
\hline & $\overline{\mathrm{PFS}}$ & $\overline{S F S}$ & $\overline{\mathrm{PFS}}$ & SFS & $\overline{\mathrm{PFS}}$ & $\overline{S F S}$ & PFS & SFS & PFS & SFS & PFS & SFS \\
\hline & 37 & 39 & 37 & 39 & 37 & 39 & 36 & 39 & 33 & 38 & 24 & 37 \\
\hline PRBC & $5 \% *$ & $28 \%$ & $27 \%$ & $26 \%$ & $14 \%$ & $10 \%$ & $6 \%$ & $10 \%$ & $3 \%$ & $5 \%$ & $0 \%$ & $3 \%$ \\
\hline Enteral Feeds & $16 \% *$ & $0 \%$ & $59 \% *$ & $28 \%$ & $84 \% *$ & $49 \%$ & $92 \%$ & $77 \%$ & $84 \%$ & $87 \%$ & $83 \%$ & $78 \%$ \\
\hline VIS & & & 3.43 & $7.76^{*}$ & & & 5.07 & 7.87 & & & $3.22^{*}$ & 6.37 \\
\hline$\geq 2$ VAD & & & $11 \%$ & $18 \%$ & & & $16 \%$ & $31 \%$ & & & $3 \%^{*}$ & $21 \%$ \\
\hline
\end{tabular}

Highest calculated vasoactive inotropic score during the first, second and third day. $\left({ }^{*} P<0.05\right)$

Abbreviations: PFS preemptive fluid strategy, SFS standard fluid strategy, PRBC packed red blood cell transfusion, VAD vasoactive drugs, VIS vasoactive inotropic score

Maintenance intravenous fluid calculation in current practices is based on the Holliday-Segar method, which was developed many decades ago. It is important to note that this method was supposed to estimate the $24 \mathrm{~h}$ water loss in hospitalized euvolemic children with normal renal function [32]. This method was not developed for use in critically ill infants, so it does not account for all the peculiarities of this group of patients. For example, febrile illness and tachypnea can increase insensible water loss. On the other hand, energy expenditure is lower in infants sedated and on MV and insensible water losses are very low in normothermic infants breathing humidified gas $[32,33]$. Based on these data, we decreased MIVF to $50 \%$ of the calculated rate while maintaining the glucose infusion rates for infants to avoid hypoglycemia.

The second element of the bundle was fluid bolus administration. In addition to the clinical assessment of hypovolemia, we added to our bundle a dynamic preload parameter, pulse pressure variation, to decide on fluid administration. Resuscitation protocols and guidelines propose an aggressive fluid management approach as the first line treatment for sepsis and shock [1, 2]. The main objective of fluid bolus administration is to correct hypovolemia, a common initial finding in pediatric sepsis and shock [34]. Currently, fluid bolus administration is a frequent intervention in the emergency department and most of the patients receive between 20 and $60 \mathrm{~mL} \cdot \mathrm{kg}^{-1}$ before PICU admission [35]. The risks and unwanted effects of this approach have been highlighted in recent studies [36-38]. Adequate fluid resuscitation increases venous return, end diastolic and systolic volume and consequently stroke volume and cardiac index. Despite the widespread clinical use, data supporting fluid bolus therapy in hospitalized critically ill children is very weak [39]. Recent adult studies have emphasized that liberal use of fluid bolus is associated with a positive fluid balance, exposing patients to the risks associated with fluid overload [24-26]. We acknowledge that the functional hemodynamic markers of preload are not widely used in critically ill children. Most devices providing invasive arterial pressure monitoring can give at least pulse pressure variation parameters with the current technology. In our view, functional hemodynamic monitoring gives to the clinician an additional parameter to assess fluid status and predict fluid responsiveness, along with vital signs, physical examinations and biomarkers of dysoxia. Only $50 \%$ of critical ill children are fluid responders [40], due to myocardial dysfunction and blunted adrenergic sensitivity $[40,41]$. Additionally, it is important to recall that fluid hemodynamic response is short. It is estimated that $85 \%$ of crystalloid fluid boluses redistribute in the interstitial tissue four hours after administration or even less in critically ill patients having an increased capillary leak $[5,42]$. In daily practice fluid boluses are the first response to multiple scenarios without a strong physiological support, i.e., tachycardia due to fever, respiratory distress or pain. With PFS, we were able to decrease fluid boluses, especially during the first $24 \mathrm{~h}$, without any clinical evidence of detrimental hypovolemia or significant renal dysfunction. Urinary output was lower in PFS, and diuretic initiation was more frequent during the first $24 \mathrm{~h}$ after admission, but renal function tests where similar between groups. Limiting unnecessary fluid administration in the PFS group was also associated with less PRBC transfusions, probably as a result of a decreased hemodilution. Vasoactive drug support was lower in the PFS group. This may seem counterintuitive, but studies in adults have shown that hemodynamic instability is associated with liberal use of fluids [43-47].

The initiation of enteral feeds occurred earlier in the PFS group. From the practical standpoint, enteral feeds allow to decrease unnecessary MIVF, improve nutritional support and are essential for the metabolic homeostasis of critically ill patient. In addition, several non-nutritive benefits of enteral feeds have been described, such as anti-inflammatory and immunomodulatory effects, which may have a major impact on the outcome of patients with ARDS [48-50]. In our center, as in many PICUs, early initiation of enteral feeds has become a priority and a quality standard. 
We found that PFS was associated with less MV days and PICU LOS, with similar findings being reported from studies in adults. Although this study was not designed to address these outcomes, we believe that these observations set the target and basis for future prospective studies in the field. A recent systematic review and meta-analysis reported that in adults and children with ARDS and sepsis, a conservative fluid strategy resulted in an increased number of ventilator-free days and a decreased length of ICU stay compared with a more liberal strategy or standard care [26].

Our study has some limitations. No data were gathered before PICU admission. The described strategy, PFS, does not apply for patients during resuscitation from septic shock. Adequate initial fluid resuscitation of patients in septic shock decreases mortality and has been well documented in many studies [1-4, 34], but the goals and metrics of these interventions need to be redefined. No specific data of the endpoints of resuscitation were registered, so it is difficult to extrapolate our protocol to unstable patients with ongoing signs of poor perfusion or dysoxia. Specifically, it is controversial what objective data the clinician must consider to terminate the resuscitation phase. All the patients included in this study suffered from sepsis due to acute pulmonary infectious diseases. Therefore, the findings in this study cannot be directly extrapolated to patients with extrapulmonary pARDS. Our measurements and outcomes were short-term and obtained upon PICU admission, so we cannot extrapolate the long-term effects using this approach. Finally, we must acknowledge the limitations of comparing our findings with a historical retrospective cohort, especially since selection bias and type II error cannot be ruled out.

\section{Conclusion}

We successfully implemented a quality improvement initiative to prevent early fluid overload in critically ill children. In our view, clinicians must be aware that a systematic approach with measures specifically limiting unnecessary fluid input (MIVF and resuscitation fluid bolus) over the first $48 \mathrm{~h}$ can be easily applied in most PICU and may prevent significant FO.

Future collaborative studies are needed to address if a preemptive fluid strategy after resuscitation from shock, in addition to optimal care for pARDS (protective mechanical ventilation, avoidance of patient ventilator asynchrony, optimal nutrition, weaning protocol, among others) might have a positive impact in outcomes.

\section{Additional file}

Additional file 1: Figure S1. Vasoactive drug use in standard fluid strategy and preemptive fluid strategy at day 1,2 and 3 of study. ${ }^{*} P<0.05$. Abbreviations: PFS: preemptive fluid strategy; SFS: standard fluid strategy. (JPG $226 \mathrm{~kb})$

\section{Abbreviations}

ARDS: Acute respiratory distress syndrome; FO: Fluid overload; ICU: Intensive care unit; IV: Intravenous; MIVF: Maintenance intravenous fluids; MV: Mechanical ventilation; pARDS: pediatric acute respiratory distress syndrome; PFO: Peak fluid overload; PFS: Preemptive fluid strategy; PRBC: Packed red blood cells; SFS: Standard fluid strategy; VAD: Vasoactive drugs; VIS: Vasoactive/inotropic score

\section{Acknowledgements}

We would like to thank Dr. Katherina Blaha for her assistance in patient screening and data collection.

\section{Funding}

This project was partially funded by Fondo Nacional de Desarrollo Científico y Tecnológico \#11160463 to FD; and \#1160631 to PC.

Availability of data and materials

IRB did not approve publication of individual data in any form.

\section{Authors' contributions}

FD designed the study, participated in patient screening, data collection and analysis, manuscript writing and edition. MN participated in patient screening, protocol design, data collection and analysis, manuscript writing and edition. PP participated in patient screening, data collection and manuscript writing. BE participated in study design, data analysis, manuscript writing and edition. PC designed the study, participated in data analysis and manuscript writing and edition. All authors read and approved the final manuscript.

\section{Competing interest}

The authors declare that they have no competing interests.

\section{Ethics approval and consent to participate}

Hospital Padre Hurtado's review board approved the quality improvement project waiving the requirement for individual written consent.

\section{Consent for publication}

Not applicable.

\section{Publisher's Note}

Springer Nature remains neutral with regard to jurisdictional claims in published maps and institutional affiliations.

\section{Author details}

${ }^{1}$ Área de Cuidados Críticos, Hospital Padre Hurtado, Santiago, Chile. ${ }^{2}$ Pediatric Intensive Care Unit, Clínica Alemana de Santiago, Santiago, Chile. ${ }^{3}$ Facultad de Medicina Clínica Alemana Universidad del Desarrollo, Santiago, Chile. ${ }^{4}$ Pediatric Intensive Care Unit, Hospital El Carmen de Maipú, Santiago, Chile. ${ }^{5}$ Centro de Investigación de Medicina Veterinaria, Escuela de Medicina Veterinaria, Facultad de Ciencias de la Vida, Universidad Andrés Bello, Avda. Republica 217, Santiago, Chile.

Received: 6 February 2018 Accepted: 21 June 2018 Published online: 26 June 2018

\section{References}

1. Carcillo JA, Kuch BA, Han YY, et al. Mortality and functional morbidity after use of PALS/APLS by community physicians. Pediatrics. 2009;124(2):500-8.

2. Davis AL, Carcillo JA, Aneja RK, et al. The American College of Critical Care Medicine Clinical Practice Parameters for hemodynamic support of pediatric and neonatal septic shock. Pediatr Crit Care Med. 2017;18(9):884-90.

3. Rhodes A, Evans LE, Alhazzani W, et al. Surviving Sepsis campaign: international guidelines for Management of Sepsis and Septic Shock: 2016. Crit Care Med. 2017:45(3):486-552.

4. Oliveira CF, Nogueira de Sá FR, Oliveira DS, Gottschald AF, Moura JD, Shibata AR, Troster EJ, Vaz FA, Carcillo JA. Time- and fluid-sensitive resuscitation for hemodynamic support of children in septic shock: barriers to the implementation of the American College of Critical Care Medicine/ Pediatric Advanced Life Support Guidelines in a pediatric intensive care unit in a developing world. Pediatr Emerg Care. 2008:24(12):810-5.

5. Hilton AK, Bellomo R. Totem and taboo: fluids in sepsis. Crit Care. 2011;15(3):164. 
6. Sampaio TZ, O'Hearn K, Reddy D, Menon K. The influence of fluid overload on the length of mechanical ventilation in pediatric congenital heart surgery. Pediatr Cardiol. 2015;36(8):1692-9.

7. Sutherland SM, Zappitelli M, Alexander SR, et al. Fluid overload and mortality in children receiving continuous renal replacement therapy: the prospective pediatric continuous renal replacement therapy registry. Am J Kidney Dis. 2010;55(2):316-25.

8. Askenazi DJ, Koralkar R, Hundley HE, Montesanti A, Patil N, Ambalavanan N. Fluid overload and mortality are associated with acute kidney injury in sick near-term/ term neonate. Pediatr Nephrol. 2013:28(4):661-6.

9. Bhaskar P, Dhar AV, Thompson M, Quigley R, Modem V. Early fluid accumulation in children with shock and ICU mortality: a matched case-control study. Intensive Care Med. 2015;41(8):1445-53.

10. Li Y, Wang J, Bai Z, et al. Early fluid overload is associated with acute kidney injury and PICU mortality in critically ill children. Eur J Pediatr. 2016;175(1):39-48.

11. Chen J, Li X, Bai Z, et al. Association of Fluid Accumulation with clinical outcomes in critically ill children with severe Sepsis. PLoS One. 2016;11(7): e0160093.

12. Hayes LW, Oster RA, Tofil NM, Tolwani AJ. Outcomes of critically ill children requiring continuous renal replacement therapy. J Crit Care. 2009;24(3):394-400.

13. Arikan AA, Zappitelli M, Goldstein SL, Naipaul A, Jefferson LS, Loftis LL. Fluid overload is associated with impaired oxygenation and morbidity in critically ill children. Pediatr Crit Care Med. 2012;13(3):253-8.

14. Foland JA, Fortenberry JD, Warshaw BL. Fluid overload before continuous hemofiltration and survival in critically ill children: a retrospective analysis. Crit Care. 2004;32(8):1771-6.

15. Diaz F, Benfield M, Brown L, Hayes L. Fluid overload and outcomes in critically ill children: a single center prospective cohort study. J Crit Care. 2017;39:209-13.

16. Cordemans C, De laet I, Van Regenmortel N, et al. Fluid management in critically ill patients: the role of extravascular lung water, abdominal hypertension, capillary leak, and fluid balance. Ann Intensive Care. 2012; 2(Suppl 1):S1.

17. Reyes-Bahamonde J, Raimann JG, Thijssen S, Levin NW, Kotanko P. Fluid overload and inflammation-a vicious cycle. Semin Dial. 2013;26(1):31-5.

18. Grams ME, Estrella MM, Coresh J, Brower RG, Liu KD. National Heart, Lung, and Blood Institute acute respiratory distress syndrome network. Fluid balance, diuretic use, and mortality in acute kidney injury. Clin J Am Soc Nephrol. 2011;6(5):966-73.

19. Murphy CV, Schramm GE, Doherty JA, et al. The importance of fluid management in acute lung injury secondary to septic shock. Chest. 2009;136(1):102-9.

20. Valentine SL, Sapru A, Higgerson RA, et al. Fluid balance in critically ill children with acute lung injury. Crit Care Med. 2012:40(10):2883-9.

21. Ingelse SA, Wösten-van Asperen RM, Lemson J, Daams JG, Bem RA, van Woensel JB. Pediatric acute respiratory distress syndrome: fluid management in the PICU. Front Pediatr. 2016:4:21.

22. Sinitsky L, Walls D, Nadel S, Inwald DP. Fluid overload at 48 hours is associated with respiratory morbidity but not mortality in a general PICU: retrospective cohort study. Pediatr Crit Care Med. 2015;16(3):205-9.

23. Flori HR, Church G, Liu KD, Gildengorin G, Matthay MA. Positive fluid balance is associated with higher mortality and prolonged mechanical ventilation in pediatric patients with acute lung injury. Crit Care Res Pract. 2011;2011:854142.

24. National Heart, Lung, and blood institute acute respiratory distress syndrome (ARDS) clinical trials network, Wiedemann HP, wheeler AP, Bernard GR, et al. comparison of two fluid-management strategies in acute lung injury. N Engl J Med. 2006:354(24):2564-75.

25. Wiedemann HP. A perspective on the fluids and catheters treatment trial (FACTT). Fluid restriction is superior in acute lung injury and ARDS. Cleve Clin J Med. 2008;75(1):42-8.

26. Silversides JA, Major $\mathrm{E}$, Ferguson AJ, et al. Conservative fluid management or deresuscitation for patients with sepsis or acute respiratory distress syndrome following the resuscitation phase of critical illness: a systematic review and meta-analysis. Intensive Care Med. 2017:43(2):155-70.

27. Pediatric Acute Lung Injury Consensus Conference Group. Pediatric acute respiratory distress syndrome: consensus recommendations from the pediatric acute lung injury consensus conference. Pediatr Crit Care Med. 2015;16(5):428-39.

28. Goldstein B, Giroir B, Randolph A. International pediatric sepsis consensus conference: definitions for sepsis and organ dysfunction in pediatrics* Pediatr Crit Care Med. 2005;6(1):2-8.
29. Blaha K, Diaz F, Quilodrán J, Medina T, Nuñez MJ, Cruces P. Safety and efficacy of a preventive strategy for fluid overload in children with sepsis and pARDS. Crit Care Med. 2016;44(12):309.

30. Mclntosh AM, Tong S, Deakyne SJ, Davidson JA, Scott HF. Validation of the vasoactive-inotropic score in pediatric Sepsis. Pediatr Crit Care Med. 2017;18(8):750-7.

31. Goldstein SL, Somers MJ, Baum MA, et al. Pediatric patients with multiorgan dysfunction syndrome receiving continuous renal replacement therapy. Kidney Int. 2005;67(2):653-8.

32. Holliday MA, Segar WE. The maintenance need for water in parenteral fluid therapy. Pediatrics. 1957;19(5):823-32

33. Raman S, Peters MJ. Fluid management in the critically ill child. Pediatr Nephrol. 2014;29(1):23-34.

34. Carcillo JA, Davis AL, Zaritsky A. Role of early fluid resuscitation in pediatric septic shock. JAMA. 1991;266(9):1242-5.

35. Long E, Babl F, Dalziel S, Dalton S, Etheridge C, Duke T, Paediatric Research in Emergency Departments International Collaborative (PREDICT). Fluid resuscitation for paediatric sepsis: a survey of senior emergency physicians in Australia and New Zealand. Emerg Med Australas. 2015;27(3):245-50.

36. Maitland K, Kiguli S, Opoka RO, et al. FEAST trial group. Mortality after fluid bolus in African children with severe infection. N Engl J Med. 2011;364(26): 2483-95.

37. Maitland K, George EC, Evans JA, et al. FEAST trial group. Exploring mechanisms of excess mortality with early fluid resuscitation: insights from the FEAST trial. BMC Med. 2013;11:68.

38. Sankar J, Ismail J, Sankar MJ, C P S, Meena RS. Fluid bolus over 15-20 versus 5-10 minutes each in the first hour of resuscitation in children with septic shock: a randomized controlled trial. Pediatr Crit Care Med. 2017;18(10):e435-45.

39. Gelbart B, Glassford NJ, Bellomo R. Fluid bolus therapy-based resuscitation for severe Sepsis in hospitalized children: a systematic review. Pediatr Crit Care Med. 2015;16(8):e297-307.

40. Gan H, Cannesson M, Chandler JR, Ansermino JM. Predicting fluid responsiveness in children: a systematic review. Anesth Analg. 2013;117(6):1380-92.

41. Lemson J, Nusmeier A, van der Hoeven JG. Advanced hemodynamic monitoring in critically ill children. Pediatrics. 2011;128(3):560-71.

42. Svensen CH, Rodhe PM, Prough DS. Pharmacokinetic aspects of fluid therapy. Best Pract Res Clin Anaesthesiol. 2009;23(2):213-24.

43. Payen D, de Pont AC, Sakr Y, et al. Sepsis occurrence in acutely ill patients (SOAP) investigators. A positive fluid balance is associated with a worse outcome in patients with acute renal failure. Crit Care. 2008;12(3):R74.

44. Mehta RL, Bouchard J, Soroko SB, et al. Program to improve Care in Acute Renal Disease (PICARD) study group: Sepsis as a cause and consequence of acute kidney injury: program to improve Care in Acute Renal Disease. Intensive Care Med. 2011:37(2):241-8.

45. Boyd JH, Forbes J, Nakada TA, Walley KR, Russell JA. Fluid resuscitation in septic shock: a positive fluid balance and elevated central venous pressure are associated with increased mortality. Crit Care Med. 2011;39(2):259-65.

46. Samoni S, Vigo V, Resendiz LI, et al. Impact of hyperhydration on the mortality risk in critically ill patients admitted in intensive care units: comparison between bioelectrical impedance vector analysis and cumulative fluid balance recording. Crit Care. 2016;20:95.

47. Sadaka F, Juarez M, Naydenov S, O'Brien J. Fluid resuscitation in septic shock the e ect of increasing fluid balance on mortality. J Intensive Care Med. 2014;29(4):213-7.

48. DeMichele SJ, Wood SM, Wennberg AK. A nutritional strategy to improve oxygenation and decrease morbidity in patients who have acute respiratory distress syndrome. Respir Care Clin N Am. 2006;12(4):547-66.

49. Wilson B, Typpo K. Nutrition: a primary therapy in pediatric acute respiratory distress syndrome. Front Pediatr. 2016;4:108

50. Jacobs BR, Nadkarni V, Goldstein B, et al. nutritional immunomodulation in children with lung injury (NICLI) study group. Nutritional immunomodulation in critically ill children with acute lung injury: feasibility and impact on circulating biomarkers. Pediatr Crit Care Med. 2013;14(1):e45-56. 